\title{
Hypoallergene Ernährung im Säuglingsalter - ist eine Prävention atopischer Erkrankungen möglich?
}

\section{Hypoallergenic Nutrition in Childhood - Is Prevention of Atopic Diseases Possible?}

\section{Autoren}

Institute

\author{
C. Bayerl', U. Winckelmann
}

Klinik für Dermatologie und Allergologie, HSK Wilhelm-Fresenius-Klinik, Städtisches Klinikum Wiesbaden

Klinik für Kinder- und Jugendmedizin, HSK Wiesbaden GmbH

\section{Bibliografie}

DOI $10.1055 / \mathrm{s}-2007-966339$

Akt Dermatol 2007; 33:

126-130 @ Georg Thieme

Verlag KG Stuttgart · New York ISSN 0340-2541

Korrespondenzadresse

Prof. Dr. med. Christiane BayerI

Klinik für Dermatologie und Allergologie

Städtische Kliniken Wiesbaden

Lehrkrankenhaus der

Universität Mainz

HSK, Wilhelm-Fresenius-Klinik

$\mathrm{GmbH}$

Aukammallee 39

65191 Wiesbaden

christiane.bayerl@hsk-

wiesbaden.de

\section{Zusammenfassung \\ $\nabla$}

In den letzten 25 Jahren hat die Zahl atopischer Erkrankungen um mehr als $150 \%$ zugenommen. Erkrankungen des atopischen Formenkreises sind seltener bei Familien mit einer höheren Geschwisterzahl, bei Kindergartenbesuch früh im Leben, bei Familien mit anthroposophischem Lebensstil, bei Kontakt mit Tieren auf dem Bauernhof in der frühen Säuglingszeit und bei hohen Endotoxinspiegeln in der Wohnung. In den ersten sechs Lebensmonaten bietet die Gabe von Brustmilch einen Schutz vor Atopie, oder aber Proteinhydrolysate, teils oder extensiv hydrolysierte Zubereitungen basierend auf Molke oder Casein und die strikte Meidung von Kuhmilch. Auf Aminosäuren aufgebaute oder sogenannte Elementardiäten werden nur bei Kindern mit nachgewiesener Kuhmilchallergie empfohlen. Beikost und feste Nahrung sollte schrittweise und erst nach dem sechsten Lebensmonat gegeben werden. Hochallergene Nahrungmittel (Ei, Milch, Soja, Fisch, Nüsse) sollten im ersten Lebensjahr bei Risikokindern gemieden werden.

\section{Epidemiologie}

$\nabla$

Jedes vierte Kind leidet an mindestens einer Manifestation einer Atopie. In Deutschland liegt die Prävalenz der atopischen Dermatitis zwischen 8-17\%. Die höchste Inzidenz der atopischen Dermatitis wird in den ersten zwei Lebensjahren erreicht. Bei mehr als der Hälfte der älteren Kinder verlieren sich die Symptome. Bei einem Elternteil mit atopischer Dermatitis liegt das Risiko eines späteren Ekzems für das Kind bei 35\%, insbesondere wenn die Mutter betroffen ist. Leiden beide Eltern an einer atopischen Dermatitis, wird das Kind ein 70\%iges Risiko einer Neurodermitis haben. Hat ein Geschwisterkind eine Neurodermitis, liegt das Risiko für das nächste Geschwister-
Meidet die Mutter während der Schwangerschaft allergene Nahrungsmittel, hat dies eher keinen Schutzeffekt in Hinsicht auf atopische Erkrankungen. Kinder mit manifester Atopie zeigen in ihrer Darmflora häufig Keime wie Bakteroides, Staphylokokkus aureus und Clostridien. Im Gegensatz dazu weisen Brustmilch-gestillte Kinder eine ähnliche Darmflora auf wie die von Kindern mit probiotischer Ernährung (Gabe apathogener Laktobazillen und Bifidusbakterien) oder auch präbiotischer Ernährung (Ernährung mit Galakto- und Fructo-Oligosacchariden, die das Wachstum von Laktobazillen und Bifidusbakterien fördern). Bei Kindern mit Atopierisiko sprechen neue Studien - allerdings noch bei spärlicher Studienlage - für probiotische Ernährung in der Schwangerschaft und bei stillenden Müttern, und präbiotische Ernährung für Kinder, die nicht gestillt werden. Zusätzliche Maßnahmen der Atopieprävention umfassen das „Nicht-Rauchen“ in Schwangerschaft, Stillzeit und in den Familien, Reduktion der Hausstaubmilben- und Schimmelpilzbelastung und keine Haustiere im späteren Kindesalter.

kind bei 35\%. In Zwillingsstudien wurde bei Homozygoten eine Konkordanz von $75 \%$ und bei Heterozygoten von 23 \% für die atopische Dermatitis gefunden $[1,2]$.

\section{Präventive Faktoren}

$\nabla$

Einen Schutz vor Atopie bietet eine hohe Geschwisterzahl, ein früher Eintritt in den Kindergarten, ein anthroposophischer Lebensstil $[3,4]$, Tierhaltung und Kontakt zu Stalltieren sowie eine hohe Endotoxin-Exposition im nahen Umfeld eines Säuglings, z.B. Bettmatratze [5-7]. Eventuell hat Hundehaltung in den ersten Lebensmonaten einen präventiven Effekt, jedoch 


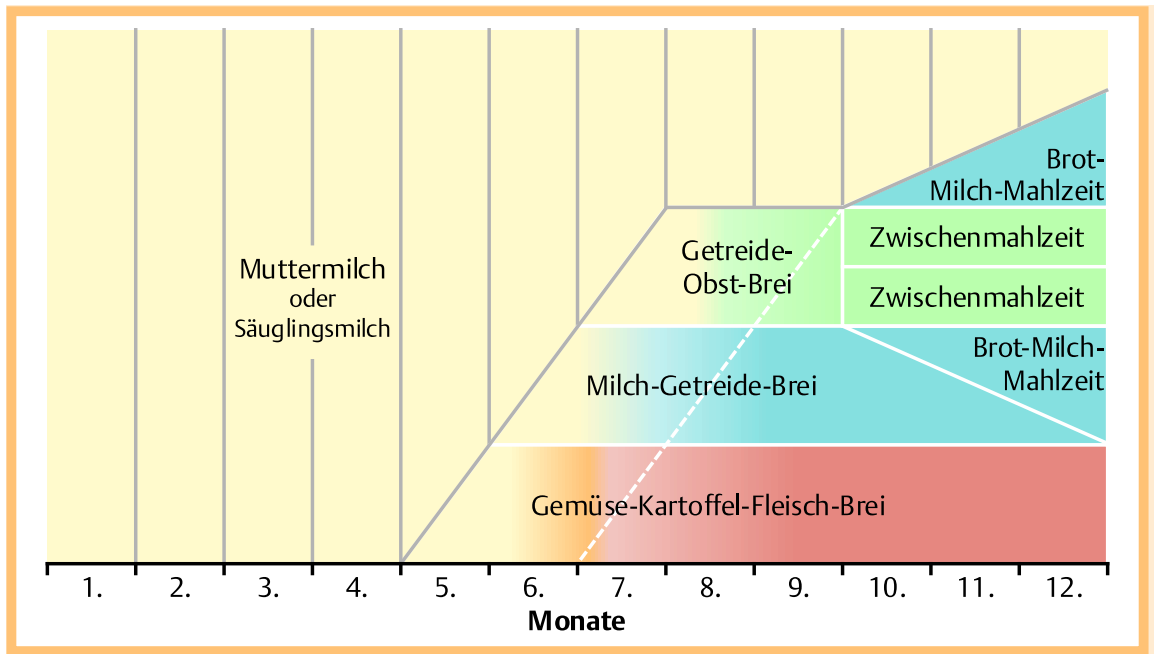

Abb. 1 Der Ernährungsplan für das erste Lebensjahr mit Beginn und Art der Beikost (Forschungsinstitut für Kinderernährung FKE/Dortmund).

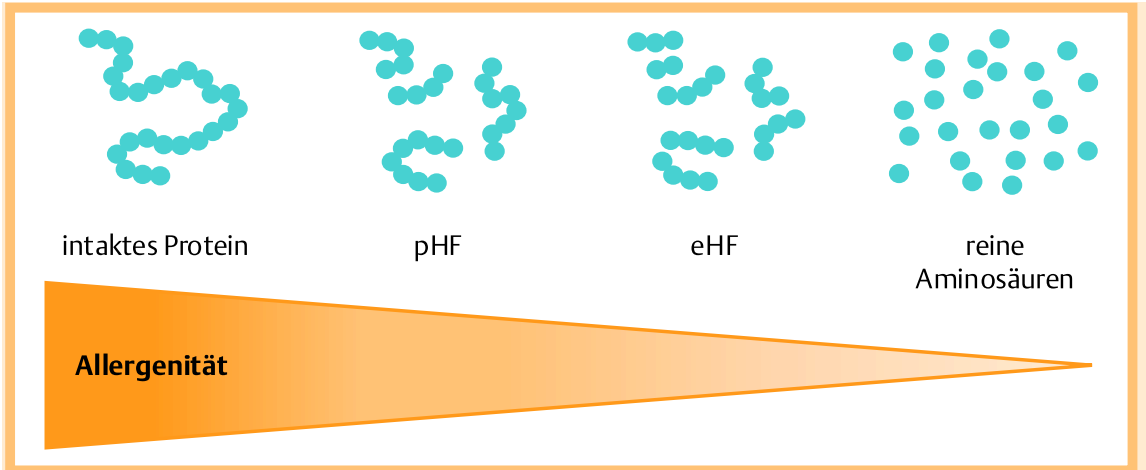

Abb. 2 Eiweiß-Hydrolysate in der hypoallergenen Säuglingsernährung, pHF = partiell hydrolysierte Formula, eHF = extensiv hydrolysierte Formula (nach Fa. Nestlé). kann daraus noch keine allgemeine Empfehlung abgeleitet werden $[5,8]$.

Zudem scheinen Mütter von Risikokindern durch Aufenthalt in ländlicher Umgebung mit Stalltieren eine Aufregulation des eigenen angeborenen Immunsystems zu erfahren, was die Kinder im Schulalter vor der Entwicklung einer atopischen Dermatitis schützt [9].

\section{Prävention durch Stillen}

Prävention erfolgt zunächst durch Stillen. Muttermilch enthält antiinfektiöse Substanzen wie sekretorisches IgA, Leukozyten und Makrophagen, antiinflammatorische Faktoren wie Lactoferrin, Lysozym und Prostaglandine sowie immunmodulierende Nukleotide und Glykoproteine, die für die Ausbildung der Mucosabarriere bedeutsam sind [10]. Lactose und Oligosaccharide finden sich als Kohlenhydrate in der Muttermilch und fördern präbiotisch den Aufbau einer Bifidusflora der Darmschleimhaut. Die Zusammensetzung der Muttermilch ist „art-eigen“ und daher günstiger als eine Molkenprotein- oder Caseinzubereitung. Andererseits finden sich aber auch Restantigenmengen, das heißt Spuren von Kuhmilcheiweiß, Erdnüssen oder was die stillende Mutter gerade genossen hat [11].

Mittlerweile zeigen mehrere Metaanalysen zur Muttermilchgabe, dass ausschließliches Stillen über 6 Monate einen präventiven Effekt auf Atopie und atopische Dermatitis hat. Die „heimliche“ Flasche im Säuglingszimmer in der Nacht sollte vermieden werden. Wichtig für den Ernährungsplan des ersten Lebensjahres ist es, Beikost nicht vor dem 4. Lebensmonat zuzugeben und neue Nahrungsmittel erst stufenweise, nach und nach zuzugeben ( $\bullet$ Abb. 1) [8,12-17]. Trotz dieser breiten Studienlage gibt es Probleme bei der Vergleichbarkeit der Studien, wie zum Beispiel die genaue Definition eines „Risikokindes“ für Atopie (Nabelschnur-IgE, positive Familienanamnese etc.), die Unmöglichkeit das Stillen zu randomisieren, die Bias durch den unterschiedlichen Lebensstil der stillenden Mütter (Rauchen, sozioökonomischer Status, Kinderzahl etc.) und die empfohlene Stilldauer, voll- oder teilgestillt, und den Beginn der Beikost.

\section{Diät der Mutter \\ $\nabla$}

Hierzu existieren verschiedene kontrollierte Studien und ein Cochrane Review. In der Schwangerschaft bietet eine allergenarme Diät keine Prävention. In der Stillzeit finden sich widersprüchliche Ergebnisse und kein sicherer Beweis für eine allergenarme Diät mit der Ausnahme von „Hochrisikokindern“ oder bereits erkrankten Kindern, die bereits eine Kuhmilchallergie aufweisen $[8,18,19]$.

\section{Eiweiß-Hydrolysate}

$\nabla$

Unterschieden werden drei Typen der Säuglingsnahrung in Hydrolysatform, die partiellen Proteinhydrolysate auf Molkebasis, die extensiven Hydrolysate auf Molke oder Caseinbasis und die Aminosäuren- oder Elementardiäten ( $\bullet$ Abb. 2, $\bullet$ Tab. 1). Ein Cochrane Review, Metaanalysen und kontrollierte Studien zeigen einen präventiven Effekt durch Hydrolysatnahrungen auf 
Tab. 1 Beispiele für Hydrolysat-Präparate, pHF = partiell hydrolysierte Formula, eHF = extensiv hydrolysierte Formula

\begin{tabular}{|c|c|c|}
\hline $\mathrm{pHF}$ & $\begin{array}{l}\text { "HA“-Nahrungen: } \\
\text { Proteinhydrolysate, } \\
\text { adaptiert auf Molkebasis }\end{array}$ & $\begin{array}{l}\text { Beba HA } \\
\text { Humana HA } \\
\text { Milumil HA }\end{array}$ \\
\hline \multirow[t]{2}{*}{ eHF } & auf Molkebasis & $\begin{array}{l}\text { Hipp HA } \\
\text { Aptamil HA } \\
\text { Alfaré }\end{array}$ \\
\hline & auf Casein-Basis & $\begin{array}{l}\text { Nutramigen } \\
\text { Pregestimil }\end{array}$ \\
\hline \multicolumn{2}{|c|}{ Aminosäure-/(Semi-)Elementardiät } & $\begin{array}{l}\text { Neocate } \\
\text { Pregomin }\end{array}$ \\
\hline
\end{tabular}

die atopische Dermatitis, meist bei der Hälfte der Studienteilnehmer und in einem höheren Prozentsatz für partiell hydrolisierte und den höchsten Prozentsatz für extensiv hydrolysierte Kost. Interessanterweise wurde im Tiermodell gezeigt, dass eine moderate Hydrolyse Toleranz induzieren kann [20]. Die Atopie-Prävention durch Hydrolysatnahrung hielt 3-5 Jahre lang an. Werden diese Ernährungsformen jedoch erst nach dem 6. Lebensmonat gestartet, gibt es keine Evidenz für einen positiven Effekt [21]. Es fehlen Studien zum direkten Vergleich zwischen gestillten und Hydrolysat-ernährten Kindern. Dies bedingt sich durch die Unmöglichkeit, Stillen zu randomisieren.

Eine Studie sei etwas detaillierter dargestellt, die GINI-Studie (German Infant Nutritional Intervention) [22,23]. Eingeschlossen wurden 2252 gesunde Neugeborene aus Westfalen und Bayern mit mindestens einem Verwandten ersten Grades mit Atopie. Die Studie war prospektiv, randomisiert und doppelblind. Alle Mütter erhielten die Stillempfehlung, dass Zufütterung erlaubt sei, wenn die Stillmenge nicht ausreiche und zwar mit herkömmlicher Säuglingsnahrung (Kuhmilchformula), partiellem Molkehydrolysat, extensivem Molkehydrolysat oder extensivem Caseinhydrolysat. Studienziel war der Vergleich zwischen verschiedenen Hydrolysatnahrungen bezüglich des präventiven Effektes auf die Entwicklung atopischer Erkrankungen im ersten Lebensjahr bei Risikokindern. 1810 Säuglinge konnten nach dem ersten Lebensjahre ausgewertet werden. 865 davon waren voll gestillt worden, 945 hatten zusätzlich eine Studiennahrung erhalten, davon insgesamt $15 \%$ ausschließlich diese Studiennahrung. Durchschnittlich war die Studiennahrung 12 Wochen lang gegeben worden, meist schon ab dem ersten Lebensmonat. Die prozentuale Inzidenz atopischer Manifestationen lag in der Kuhmilchgruppe bei 15,6\%, der Gruppe mit partiellem Molkehydrolysat bei 10,8\%, der Gruppe mit extensivem Molkehydrolysat bei $14,3 \%$ und bei der Gruppe mit extensivem Caseinhydrolysat bei 9,1\%, wobei letzte Gruppe im Unterschied signifikant war. Die prozentuale Inzidenz der atopischen Dermatitis lag in der Kuhmilchgruppe bei 14,8\%, der Gruppe mit partiellem Molkehydrolysat bei 9,1\%, der Gruppe mit extensivem Molkehydrolysat bei 13,0\% und bei der Gruppe mit extensivem Caseinhydrolysate bei 7,3\%, wobei die Gruppen mit partiellem Molkehydrolysat und die mit extensivem Caseinhydrolysat statistisch signifikante Unterschiede zeigten. Bei Sichtung der Inzidenzen der Säuglinge mit atopischer Dermatitis in der Familienanamnese, lag die Inzidenz für die Gruppe mit extensiven Caseinhydrolysaten zwar am niedrigsten, war jedoch nicht signifikant.

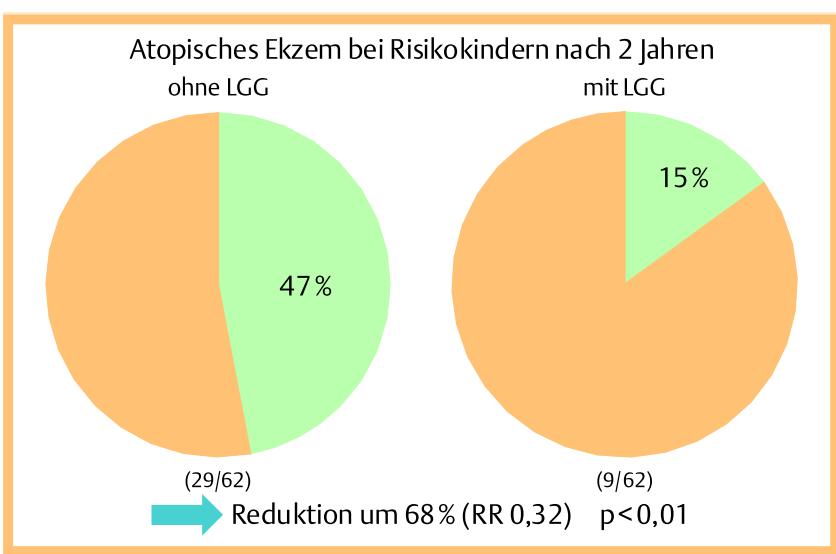

Abb. 3 Inzidenz der atopischen Dermatitis bei Risikokindern nach 2 Jahren im Vergleich mit und ohne probiotische Ernährung, gegeben 2-4 Wochen vor Geburt und in der Stillzeit.

\section{Probiotika}

$\nabla$

Ziel einer pro- und präbiotischen Ernährung ist es, eine Darmflora dominiert von Bifidusbakterien und Laktobazillen zu erreichen, wie es bei mehr als 80\% der gestillten Säuglinge der Fall ist. Flaschenernährte Kinder entwickeln diese Flora nur in 30-40\%. Kinder mit atopischer Dermatitis haben signifikant häufiger Bakteroides, Staphylokokkus aureus und Clostridien in ihrer Darmflora [24,25].

Probiotika sind definiert als apathogene Mikroorganismen, die der Nahrung beigefügt werden und durch Verbesserung der Darmflora die Gesundheit günstig beeinflussen sollen, z.B. Lactobacillus GG/Lactobacillus rhamnosus oder Bifidusbacterium lactis. Probiotika sind resistent gegen Magensaft und Verdauungsenzyme. Eine probiotisch kolonisierte Mukosa vermindert die Adhärenz und das Wachstum pathogener Keime. Die Mukosabarriere ist verbessert und die IgA-Bildung wird angeregt. Dies senkt die Permeabilität der Mukosa und reduziert die Sekretion von Entzündungsmediatoren (TGF- $\beta$ und IL-10). In einer doppelblinden, randomisierten, plazebokontrollierten Studie erhielten Schwangere mit hohem Atopierisiko ein Lactobacillus-Probiotikum 2-4 Wochen vor Geburt bis zum Abstillen und anschließend die Kinder selbst bis zum 6. Lebensmonat. Ziel war es, die Atopieprävalenz der Kinder mit 2 Jahren zu erheben. Eingeschlossen wurden 64 Mutter-Kind-Paare [26,27]. Es konnte eine Reduktion der atopischen Dermatitis bei Risikokindern um $49 \%$ erzielt werden. In einer weiteren doppelblinden, randomisierten, plazebokontrollierten Studie wurde ein Lactobacillus-Probiotikum an Schwangere mit hohem Atopierisiko gegeben, wiederum 2-4 Wochen vor Geburt und bis zum Abstillen, aber diesmal nicht an die Säuglinge selbst. Wiederum wurde die Prävalenz des atopischen Ekzems mit 2 Jahren erhoben und die TGF- $\beta$-Konzentration in der Muttermilch bei 62 Mutter-Kind-Paaren. Es zeigte sich eine Reduktion des atopischen Ekzems bei Risikokindern nach 2 Jahren um $68 \%$ und eine Reduktion des TGF- $\beta$-Spiegels [28] ( $\bullet$ Abb. 3).

Weiter wurde der therapeutische Effekt von Hydrolysat alleine, Hydrolysat plus Bifidusbakterien und Hydrolysat plus Laktobazillen bei bisher voll gestillten Säuglingen untersucht. Nach zwei Monaten war das Ekzem schwächer ausgeprägt in den Gruppen mit Probiotika [29]. In einer doppelblinden, plazebokontrollierten Cross-over-Studie fand sich eine signifikant gerin- 


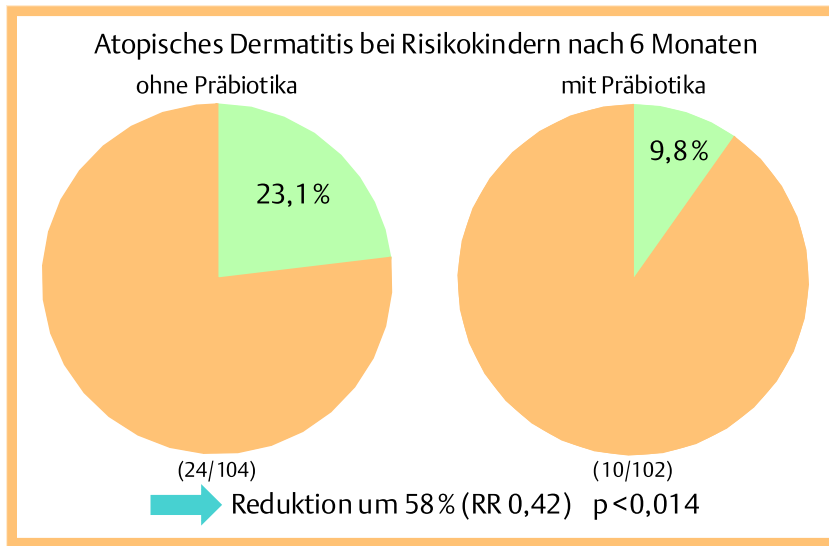

Abb. 4 Inzidenz der atopischen Dermatitis bei Risikokindern nach 6 Monaten im Vergleich mit und ohne präbiotische Ernährung, gegeben von der Geburt bis zum Ende des 4. Monats.

gere Ausdehnung des atopischen Ekzem nach 6 Wochen Probiotika-Gabe [30].

Als mögliche Nachteile der Gabe von Probiotika werden aktuell die Übertragung von Resistenzgenen, die Bildung biogener Amine, die Aktivierung von Prokanzerogenen, die Dekonjugation von Gallensalzen und eine Thrombozytenaggregationssteigerung diskutiert [31]. Dagegen konnte gezeigt werden, dass der Effekt von Impfungen durch Probiotika nicht negativ beeinflusst wird [32].

\section{Präbiotika}

$\nabla$

Präbiotika sind definiert als nicht verdauliche Lebensmittelbestandteile (Oligosaccharide), die das Wachstum und die Kolonisation von probiotisch wirksamen Bakterien im Darm fördern und dadurch die Gesundheit verbessern sollen, z.B. Galaktound Fructo-Oligosaccharide. Präbiotika sind resistent gegen die Verdauungsenzyme des Magensaftes und werden selektiv durch Laktobazillen und Bifidusbakterien verstoffwechselt. Dies führt zu einer pH-Absenkung des Stuhls durch Fermentierung zu kurzkettigen Fettsäuren mit einer trophischen Wirkung auf die Mukosa, bzw. der Bildung von Laktat/Azetat, was einen Schutz vor pathogenen Keimen bietet [33].

In einer doppelblinden, randomisierten, plazebokontrollierten Studie wurde Hydrolysat mit Zusatz von Präbiotika (Verum) versus Hydrolysat mit Maltodextrin (Plazebo) an Neugeborene mit hohem Atopierisiko unmittelbar nach Geburt bis zum Ende des vierten Monats gegeben. Beurteilt wurde die Inzidenz des atopischen Ekzems mit 6 Monaten bei 104 Mutter-Kind-Paaren. Es zeigte sich bei Risikokindern eine Reduktion der Inzidenz der atopischen Dermatitis um 58\% [34] ( Abb. 4).

\section{Schlussfolgerung}

$\nabla$

Nach den Ergebnissen der Konsensuskonferenz zur Atopie-Prävention, die in Leitlinien mündeten, gilt der höchste Evidenzgrad und daher auch die Empfehlungsklasse A für 4-6 Monate Stillen und „Nicht-Rauchen“ [8]. Eine Diät der Mutter in der Schwangerschaft ist nicht nötig. Kann nicht gestillt werden oder nur teilweise, sollte eine der überprüften Hydrolysatnahrungen in den ersten 6 Monaten gegeben werden. Gut belegt und daher Emp- fehlungsklasse B ist das Meiden der Flasche „zwischendurch“. An sich ist in der Stillzeit keine Diät der Mutter notwendig, es sei denn es handelt sich um ein „Hochrisikokind“ oder ein Kind, bei dem eine Kuhmilchallergie bereits manifest ist. Bei Risikokindern sollte stark allergene Beikost wie Ei, Kuhmilch, Soja, Fisch und Nüsse erst nach dem 12. Lebensmonat gegeben werden. Sicherlich ist es in diesen Fällen auch sinnvoll, eine Beikost erst ab dem 6. Lebensmonat zu geben. Probiotika für Schwangere und Stillende mindern das Risiko der Atopieentwicklung und sind auch effektiv bei nicht gestillten Kindern. Dagegen sollten Elementar- oder Aminosäurenahrungen nur bei gesicherten Nahrungsmittelallergien gegeben werden.

Offen bleiben in der alimentären Atopie-Prävention die Fragen, ob Hydrolysatnahrung nach dem 6. Monat überhaupt noch einen Effekt hat und ob Nicht-Risikokinder auch von präventiven Maßnahmen profitieren. Über die alimentären Präventionsmöglichkeiten für Riskokinder hinaus bleiben die Empfehlungen „Nicht-Rauchen“, Hausstaubmilben- und Schimmelpilz-Belastung reduzieren und keine Haustiere in späteren Lebensmonaten anschaffen [8].

\section{Abstract}

\section{Hypoallergenic Nutrition in Childhood - Is Prevention of Atopic Diseases Possible? (s)}

In the last 25 years, atopic diseases have increased by more than $150 \%$. Atopy occurs less frequently in families with a higher number of siblings, in cases of early admittance to kindergarden, in families with anthroposophic lifestyles, in cases of early contact with farm animals and high endotoxin levels in the immediate environment.

In the first six months of life, nutritional protection is provided by breast feeding, protein hydrolysates such as partially or extensively hydrolysed formula based on whey or caseine and strict avoidance of cow's milk. Amino acids or elementary diets are recommended only in children with proven milk protein allergy. Supplementary diet and solid food should be started step by step after the sixth month and high allergenic food (egg, milk, soy, fish, nuts) must be avoided in the first year. During pregnancy, avoidance of potential allergens by the mother has not been proven to prevent atopic diseases. In children with allergy, bacteroides, staphylococcus aureus and clostridiae are often resident in the gut mucosa. In contrast, probiotic nutrition (apathogenic lactobacilli or bifidobacteria) or praebiotic nutrition (galacto- or fructo-oligosaccharides favoring the growth of lactobacilli and bifidobacteriae) result in an intestinal flora such as is found in breast-fed children. For children at risk of allergy, recent studies - still only few and therefore preliminary - argue in favour of probiotics for pregnant and breast-feeding women and praebiotics for non breast-fed children.

Additional measures for atopy avoidance are not smoking in pregnancy, during breast feeding and in families with a child at risk, reduction of housedust mites and moulds and no pets in later childhood. 


\section{Literatur}

1 Weißbuch Allergie in Deutschland, 2. Auflage. München: Urban \& Vogel Medien und Medizin Verlagsgesellschaft mbH \& KG, 2004: 19-48

2 Illi S, von Mutius E, Lau S, Nickel R, Gruber C, Niggemann B, Wahn U Multicenter Study Group. The natural course of atopic dermatitis from birth to age 7 years and the association with asthma. J Allergy Clin Immunol 2004; 113: 925 - 931

3 Alm JS, Swartz J, Lilja G, Scheynius A, Pershagen G. Atopy in children of families with an anthroposophic lifestyle. Lancet 1999; 353: 14851488

4 Alfven T, Braun-Fahrländer C, Brunekreef B, von Mutius E, Riedler J et al. Allergic diseases and atopic sensitization in children related to farming and anthroposophic lifestyle - the PARSIFAL study. Allergy 2006; 61: $414-421$

5 Campo P, Kalra HK, Levin L, Reponen T, Olds R, Lummus ZL et al. Influence of dog ownership and high endotoxin on wheezing and atopy during infancy. J Allergy Clin Immunol 2006; 118: 1271 - 1278

6 Riedler I, Braun-Fahrländer C, Eder W, Scheurer M, Wasner M, Maisch S Alex study team. Exposure to farming in early life and development of asthma and allergy: a cross-sectional survey. Lancet 2001; 358: $1129-1133$

7 Braun-Fahrländer C. Environmental exposure to endotoxin and other microbial products and the decreased risk of childhood atopy: evaluating developments since April 2002. Curr Opin Allergy Clin Immunol 2003; 3: 325-329

8 AWMF-Leitlinie Allergieprävention. Allergo Journal 2004; 13: 252 260

9 Ege MJ, Bieli C, Frei R, von Strien RT, Riedler I et al. Parsifal Study team. Prenatal farm exposure is related to the expression of receptors of the innate immunity and to atopic sensitization in school-age children. J Allergy Clin Immunol 2006; 117: 817-823

10 Bayerl C, Voigtländer V. Stillen - Prophylaxe einer Neurodermitis? Z Allg Med 1992; 68: $342-348$

11 Bayerl C, Voigtländer V. Muttermilch - trotz Umweltbelastung eine Prophylaxe der Neurodermitis? Therapeutikon 1993; 7: 91 -96

12 Kramer $S$. Does breast feeding help protect against atopic disease? Biology, methodology, and a golden jubilee of controversy. J Pediatr 1988; $112: 181-190$

13 Saarinen UM, Kajosaari M. Breastfeeding as prophylaxis against atopic disease: prospective follow-up study until 17 years old. Lancet 1995; 346: 1065 - 1069

14 Fergusson DM, Horwood IJ, Shannon FT. Early solid feeding and recurrent childhood eczema: a 10-year longitudinal study. Pediatrics 1990; 86: $541-546$

15 Gdalevich M, Mimouni D, David M, Mimouni M. Breast-feeding and the onset of atopic dermatitis in childhood: a systematic review and meta-analysis of prospective studies. J Am Acad Dermatol 2001; 45: $520-527$

16 Benn CS, Wohlfahrt I, Aaby P, Wetgaard T, Benfeldt E, Michaelsen KF et al. Breastfeeding and risk of atopic dermatitis by parental history of allergy, during the first 18 months of life. Am J Epidemiol 2004; 160: $217-223$

17 Schoetzau A, Filipiak-Pittroff B, Franke K, Koletzko S, von Berg A, Grubl A et al. Effect of exclusive breast-feeding and early solid food avoidance on the incidence of atopic dermatitis in high-risk infants at 1 year of age. Pediatr Allergy Immunol 2002; 13: 234-242
18 Lilja G, Dannaeus A, Falth-Magnusson K, Graff-Lonnevig V, Johansson SG, Kjellman NI, Oman H. Immune response of the atopic woman and foetus: effects of high-and low-dose allergen intake during late pregnancy. Clin Allergy 1988; 18: 131 - 143

19 Zeiger RS. Dietary manipulations in infants and their mothers and the natural course of atopic disease. Pediatr Allergy Immunol 1994; 5: $33-43$

20 Schoetzau A, Gehring U, Wichmann HE. Prospective cohort studies using hydrolysed formulas for allergy prevention in atopy-prone newborns: a systematic review. Eur J Pediatr 2001; 160: 323-332

21 AWMF-Leitlinie Vorgehen bei vermuteter Nahrungsmittelallergie bei atopischer Dermatitis. Allergo Journal 2002; 11: 386-393

22 Von Berg A, Koletzko S, Filipiak-Pittroff B, Laubereau B, Grubl A, Wichmann HE. Certain hydrolyzed formulas reduce the incidence of atopic dermatitis but not that of asthma. Three-Year results of the German Infant Nutritional Intervention Study. J Allergy Clin Immunol 2007; 19: in press

23 Von Berg A, Koletzko S, Grubl A, Filipiak-Pittroff B, Wichmann HE, Bauer $C P$, Reinhardt $D$ et al. The effect of hydrolyzed cow's milk formula for allergy prevention in the first year of life: the German Infant Nutritional Intervention Study; a randomized double-blind trial. J Allergy Clin Immunol 2003; 111: 533-540

24 Woodcock A, Moradi M, Smillie FI, Murray CS, Burnie JP, Custovic A. Clostridium difficile, atopy and wheeze during the first year of life. Pediatr Allergy Immunol 2002; 13: 357-360

25 Murray CS, Tannock GW, Simon MA, Harmsen HI, Welling GW, Custovic $A$, Woodcock A. Fecal microbiotia in sensitized wheezy and non sensitized non-wheezy children: a nested case-control study. Clin Exp Allergy 2005; 35: $741-745$

26 Kalliomaki M, Salminen S, Poussa T, Arvilommi H, Isolauri E. Probiotics and prevention of atopic desease; 4-year follow-up of a randomised placebo-controlled trial. Lancet 2003; 361: 1869-1871

27 Kalliomaki M, Salminen S, Arvilommi H, Kero P, Koskinen P, Isolauri E. Probiotics in primary prevention of atopic disease: a randomised placebo-controlled trial. Lancet 2001; 357: 1076-1079

28 Rautava S, Kalliomaki M, Isolauri E. Probiotics during pregnancy and breast-feeding might confer immunomodulatory protection against atopic disease in the infant. J Allergy Clin Immunol 2002; 109: 119121

29 Isolauri E, Arvola T, Sutas Y, Moilanen E, Salminen S. Probiotics in the management of atopic eczema. Clin Exp Allergy 2000; 31: 1640-1610

30 Rosenfeldt V, Benfeldt E, Nielses SD, Michaelsen KF, Jeppesen DL, Valerius $\mathrm{NH}$, Paerregaard A. Effect of probiotic Lactobacillus strains in children with atopic dermatitis. J Allergy Clin Immunol 2003; 111: 389- 395

31 Macfarlane GT, Cummings JH. Probiotics, infection and immunity. Curr Opin Infect Dis 2002; 15: $501-506$

32 Kukkonen K, Nieminen T, Poussa T, Savilahti E, Kuitunen M. Effect of probiotics on vaccine antibody responses in infancy - a randomized placebo-controlled double-blind trial. Pediatr Allergy Immunol 2006; 17: $416-421$

33 Haarman M, Knol J. Quantitative real-time PCR analysis of fecal Lactobacillus species in infants receiving a prebiotic infant formula. Appl Environ Microbiol 2006; 72: 2359-2365

34 Moro G, Arslanoglu S, Stahl B, Jelinek I, Wahn U, Boehm G. A mixture of prebiotic oligosaccharides reduces the incidence of atopic dermatitis during the first six months of age. Arch Dis Child 2006; 91: 814-819 